\title{
LEARNING-BASED SEGMENTATION OF OPTIC DISC IN RETINAL IMAGES USING CLUSTERING TREES AND LOCAL MODE FILTERING
}

\author{
Michael Osadebey, Marius Pedersen and Dag Waaler \\ Norwegian University of Science and Technology, Norway
}

\begin{abstract}
Delineation of the optic disc boundary in retinal images is the first step towards the computation of cup-to-disc ratio, an important indicator of ophthalmic pathologies such as glaucoma. This paper proposes the combination of learning-based clustering trees with local mode filtering for the segmentation of the optic disc region in retinal images. The algorithm identifies candidate optic disc region by extracting and pooling low-level features at different clustering resolutions from the filtered region-of-interest in two color channels. Thereafter, we use learned geometric properties such as area, eccentricity and solidity to extract high-level features for the identification of connected components, which most likely belong to the optic disc region. The final stage pools and fully connects these connected components into a single segmented region. Performance evaluation on three publicly available datasets from IDRID, DRISHTI-GS and MESSIDOR demonstrate promising results that are comparable to state-of-the-art algorithms.
\end{abstract}

\section{KEYWORDS}

Glaucoma, Retinal Images, Optic Disc, Clustering Trees, Local Mode Filtering

\section{INTRODUCTION}

The cup-to-disc ratio, defined as the diameter of the optic cup divided by the diameter of the optic disc, is one of several metrics used by ophthalmologists to assess the progression of glaucoma disease. The first step in the computation of cup-to-disc ratio is delineation of the boundaries covered by the optic disc and optic cup. Manual delineation in large-scale retina screening is time consuming, cumbersome and prone to fatigue-induced errors. Assessments from different experts can be subjective with high intra-reader and inter-reader variability. Therefore, automated methods are preferred because it is efficient and reproducible.

Prior work on optic disc segmentation combine several techniques, however, they can be broadly classified as template-based, deformable models and machine learning. Template-based methods exploit the generally accepted oval-shaped structure of the optic disc as well as its color and appearance. Aquino et al. (2010) identifies a region-of-interest where the optic disc region is located within the image. Next is the extraction of the optic disc boundary from the red and green channels by means of morphological operation and edge detection. The optic disc boundaries from both channels are then approximated using the circular Hough transform. Roychowdhury et al. (2015) combine thresholding and morphological reconstruction of the red color channel with region-based features and a Gaussian mixture model classifier to identify candidate optic disc region. The candidate optic disc region having the best-fit ellipse is regarded as the segmented optic disc.

Approaches based on deformable models generally adopt a template-matching technique to localize the optic disc region, followed by the use of a deformable model for segmentation of the optic disc boundaries. Lowell et al. (Lowell et al., 2004) localizes the disc region using a template in the form of a Laplacian of Gaussian filter. Segmentation of the optic disc is based on a global elliptical model and a local deformable model. Dey et al. (2019) localize the optic disc region before the application of an affine transformation-based snake model in a gradient vector field. The gradient descent technique was used to determine the initial parameters of the affine transformation. The snake evolves to enclose the desired boundary by calculating the partial derivatives and updating the affine transformation parameters. 
In the past decade, there has been growing interest in applying machine learning to the segmentation of optic disc. Lim et al. (2015) proposed the fine-net convolutional neural network architecture, a variant of full-resolution residual networks (Pohlen et al., 2017) designed for semantic segmentation. The method begins with a preprocessing stage to remove blood vessels and localize a square region around the optic disc. Thereafter, following classification by a convolutional neural network, pixel-level probability maps of the optic disc, optic cup and background are generated. The predicted optic disc and cup boundaries are obtained after postprocessing the pixel-level probability maps.

This paper proposes a new approach to segment optic disc in retinal images. Three key contributions are: Contribution 1: The application of clustering tree that overcomes the limitations of $\mathrm{k}$-means clustering for segmentation of optic disc. Clustering tree is clustering at multiple clustering resolutions (Zappia et al., 2018).

Contribution 2: We exploit the noise reducing capability of the mode filter to reduce the effect of occlusion by blood vessels within the optic disc region.

Contribution 3: Feature extraction and pooling from two color channels to optimize segmentation.

\section{MATERIALS AND METHODS}

This study utilize three publicly available retinal fundus images from the Indian Diabetic Retinopathy Image Dataset (IDRID) (Porval et al., 2018, https://idrid.grand-challenge.org/\}, the DRISHTI-GS dataset (Sivaswamy et al., 2014, https://cvit.iiit.ac.in/projects/mip/drishti-gs/mip-dataset2/enter.php), and the MESSIDOR consortium (Klein et al, 2016, http://www.adcis.net/en/third-party/messidor/.). The IDRiD database was part of 'Diabetic Retinopathy: Segmentation and Grading Challenge' workshop at IEEE International Symposium on Biomedical Imaging (ISBI-2018). It consists of two separate sets of images. The first set has 516 images (413 training and 103 testing) with manually marked OD center and fovea location. The second set that has 81 images (54 training and 27 testing) having manually marked optic disc ground truth for both the training and testing images and dimension $4288 \times 2848$ pixels. In this study, we utilized the second set. The DRISHTI-GS dataset consists of 50 images with resolution $2896 \times 1944$ and was downloaded from GitHub website (https://github.com/seva100/optic-nerve-cnn). The MESSIDOR database consists of 1200 retinal fundus images having three different resolutions of $1140 \times 960,2240 \times 1488$ and $2304 \times 1536$. The manually segmented optic disc rim for the MESSIDOR dataset is available at (Huelva, 2012, http://www.uhu.es/retinopathy).

\subsection{Methodology}

The four phases in our methodology are data preparation, learning, preprocessing and algorithm design.

\subsubsection{Data Preparation}

All datasets were manually divided into two categories; oculus sinister (left eye) and oculus dexter (right eye). They were further divided into learning and testing data. The learning data consist of 113 oculi sinister and 113 oculi dexter. The distribution of the learning data are 16, 10 and 200 images from IDRID, DRISHTI-GS and MESSIDOR, respectively. Corresponding distribution of the testing data are 65, 40 and 1000. The ground truth of oculus sinister and oculus dexter were separately summed. The major axis length of the summed image was computed and used to build separate region-of-interest template for each category. Since images from the three publicly available databases had different resolutions, we standardize these images by cropping the region-of-interest, without loss of image resolution, to a dimension of $850 \times 850$. 


\subsubsection{Learning}

In this phase, we extract and analyze features from learning data to determine the algorithm operating parameters. Five region properties of the ground truth for each learning data was computed. They are area, eccentricity, solidity, major axis length and minor axis length. Using the k-means clustering algorithm (Lloyd et al., 1982\}, we partition the red and green channels of each image into $\mathrm{k}=(1,2, \ldots, 8)$ predefined number of clusters. For each clustered image, clusters within the optic disc region were extracted and their region properties were computed. Region properties from the ground truth and the clustered images were analyzed using histogram to determine the algorithm operating parameters displayed in Table 1.

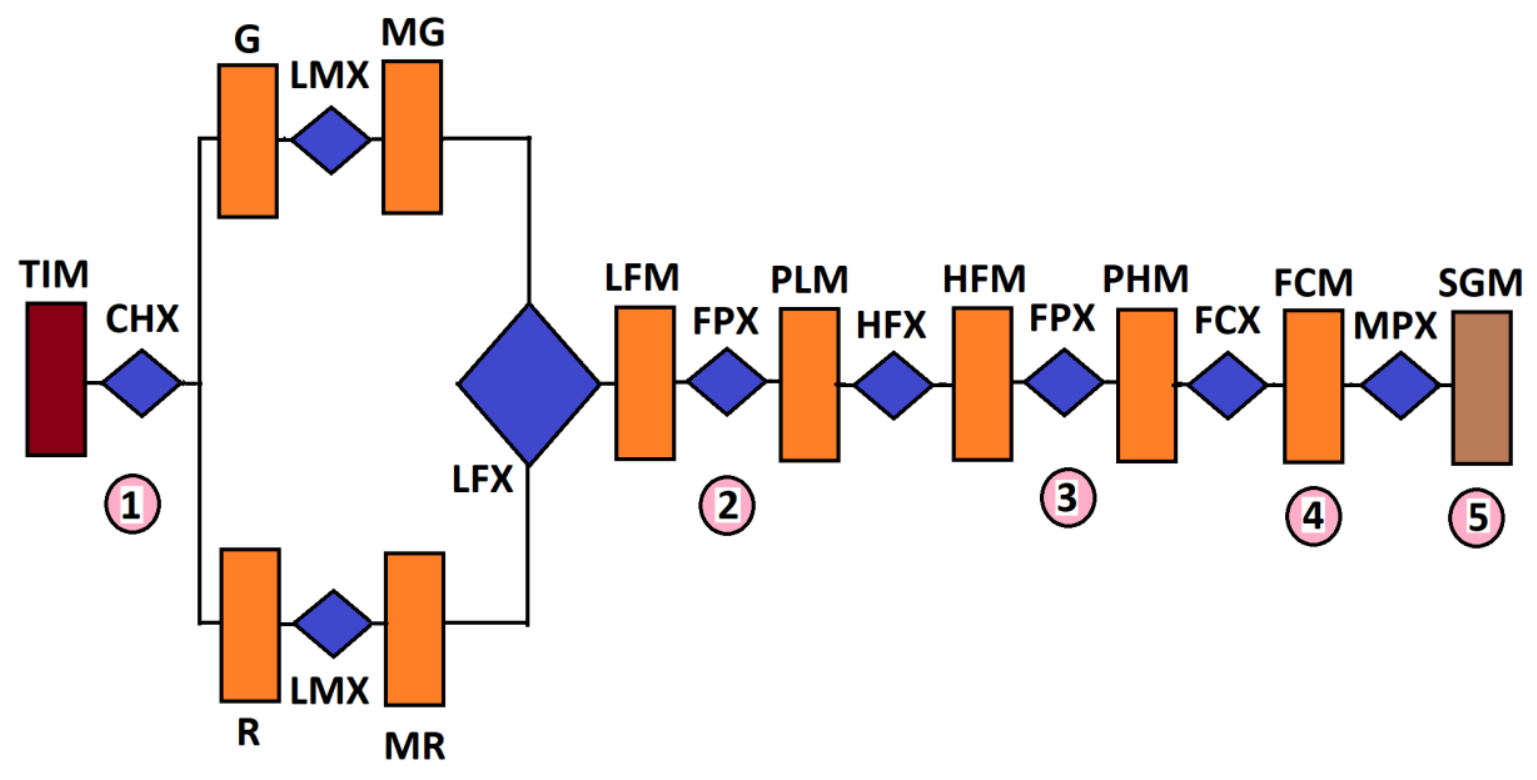

Figure 1. Flow chart of the five successive steps to implement the proposed method

Table 1. Algorithm operating parameters (in pixels) as determined from the learning phase of our methodology

\begin{tabular}{|l|l|l|l|l|l|}
\hline $\begin{array}{l}\text { Operating } \\
\text { Parameters }\end{array}$ & Area & Eccentricity & Solidity & $\begin{array}{l}\text { Minor Axis } \\
\text { Length }\end{array}$ & $\begin{array}{l}\text { Major Axis } \\
\text { Length }\end{array}$ \\
\hline $\begin{array}{l}\text { Minimum } \\
\text { Value }\end{array}$ & 10000 & 0 & 0.3 & 60 & 60 \\
\hline $\begin{array}{l}\text { Maximum } \\
\text { Value }\end{array}$ & 60000 & 0.7 & 1.0 & 300 & 300 \\
\hline
\end{tabular}

For each learning data, pixel intensity levels of the red and green channels were resealed to lie between 0 and 255. Thereafter, each image was partitioned into distinct blocks of size $45 \mathrm{x} 45$. The maximum variance computed from the blocks for each image were analyzed using histogram. The minimum and maximum values of the variance are the parameters we use to scale the size of the mode filter from $7 \times 7$ to $45 \times 45$. The red and green channels typically have low and high variance, respectively.

\subsubsection{Preprocessing}

Our recent work (Osadebey et al., 2019) was adapted for the evaluation of contrast quality attribute in retinal images. A contrast quality score of 0.45 was fixed as the acceptable limit of quality score. Images with quality scores below the acceptable limits were enhanced by correction for illumination, using the method proposed in (Marrugo et al., 2015\} to improve their quality. 


\subsubsection{Algorithm Design}

The flow chart in Figure 1 describes the five successive steps through which the proposed method segments a typical color fundus image shown in Figure 2 a to extract optic disc region shown in Figure $2 \mathrm{f}$.

\section{Step 1: Local Mode Filtering}

From the image (TIM) shown in Figure 2a, we extract $(\mathrm{CHX})$ the red $(\mathrm{R})$ and green $(\mathrm{G})$ color channels shown in Figure 2b-c, respectively. A duplicate copy of each image was partitioned into distinct blocks of size $45 \times 45$. Based on the maximum variance recorded from the blocks, the original red and green channel images were convolved with mode filter that varies from $7 \times 7$ and $45 \times 45$. The mode filter (LMX) was implemented using a sliding neighborhood operation. Local mode filter, like the median filter, is a nonlinear filter which reduces noise and potentially reduce occlusion by blood vessels. Figure $2 \mathrm{~d}$-e show the red and green color channels after filtering with a mode filter.

\section{Step 2: Low Level Feature Extraction}

The filtered red and green color channels undergo 8 successive k-means clustering operations. Figure 3b-g show low-level feature images (LFM) generated from 6 successive clustering operations, that is, $\mathrm{k}=(3,4,5,6,7,8)$ predefined number of clusters. In Figure $3 \mathrm{~b}-\mathrm{g}$, it is evident that some pixels change clusters as the clustering resolution increases. Of a particular note is the red-colored cluster which gradually shrink in size until it stabilizes at the optic disc region. The mean pixel intensity of clusters in each feature image are sorted in ascending order. We carry out feature pooling operation (PLM) by thresholding the clustered image. The threshold is set at pixel label with maximum intensity level (in red color). This gives the pooled low-level feature image (PLM) (not displayed). The pooled features from all the clustered image is a binary image with dimension $850 \times 850 \times \kappa$ where $\kappa$ is the clustering resolution.



(a)

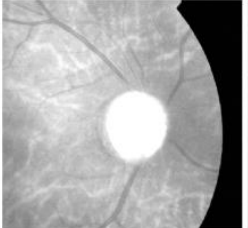

(b)

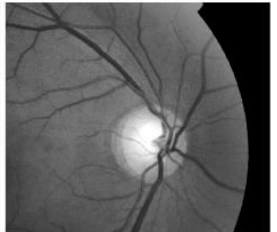

(c)

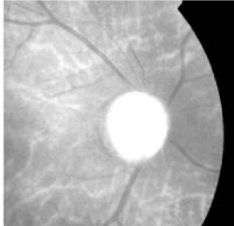

(d)



(e)

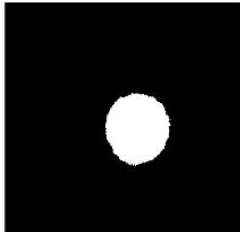

(f)

Figure 2. (a) Original color fundus image (b) Red color channel (c) Green color channel (d) Red colored channel in (b) filtered by a 7 x 7 mode filter (e) Green colored channel in (c) filtered by a 45 x 45 mode filter (f) Segmented optic disc region



(a)

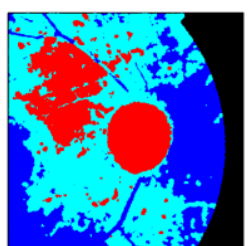

(b)

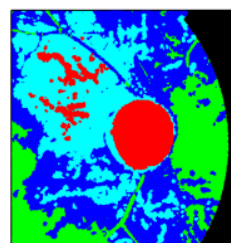

(c)

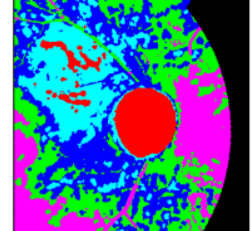

(d)

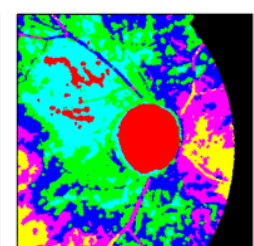

(e)

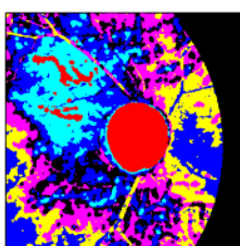

(f)

Figure 3. (a) The low-level feature images generated using k-means-based clustering tree at predefined number of clusters (a) $\mathrm{k}=3$, (b) $\mathrm{k}=4$, (c) $\mathrm{k}=5$, (d) $\mathrm{k}=6$, (e) $\mathrm{k}=7$ and (f) $\mathrm{k}=8$. The red-colored cluster, which describes possible optic disc location, gradually shrink in size with increasing predefined number of clusters until it stabilizes at the optic disc region

\section{Step 3: High Level Feature Extraction}

Region properties values derived during the learning phase are the thresholds we use for filtering the pooled low-level features (PLM) to extract (HFX) high-level feature image (HFM) (not displayed) that most likely contain the optic disc region. Feature pooling operation (FPX) is executed on the high-level feature image (HFM) by summing along the third dimension, resulting in a non-binary image which we refer to as pooled high level feature image (PHM) (not displayed) of dimension 850 x 850 . 


\section{Step 4: Fully Connected Layer}

The pooled high-level feature image from the red and green channels are fully-connected (FCX) by pixel-wise summation. Thereafter, we set a global threshold at zero-pixel intensity to obtain a binary image which we term as fully connected layer image (FCM) (not displayed).

\section{Step 5: Segmented Object}

In this final step, we eliminate false positives such as bright lesions that may have been segmented alongside the optic disc. Eccentricity region property of the fully connected layer image (FCM) is computed to extract connected component with maximum eccentricity. Thereafter, a hole filling operation (MPX) is executed to produce the segmented optic disc (SGM) shown in Figure 2f.

\section{RESULTS}

Table 2. Performance evaluation results recorded by proposed method and results published for four state-of-the-art algorithms on IDRID dataset. (-) indicates result not available

\begin{tabular}{|c|c|c|c|c|c|}
\hline Metric & $\begin{array}{c}\text { Dey et al. } \\
(\mathbf{2 0 1 9})\end{array}$ & $\begin{array}{c}\text { Porval al. } \\
\mathbf{( 2 0 1 8 )}\end{array}$ & $\begin{array}{c}\text { Chalakkal et al. } \\
\mathbf{( 2 0 1 8 )}\end{array}$ & $\begin{array}{c}\text { Baid et al. } \\
\mathbf{( 2 0 1 9 )}\end{array}$ & Proposed \\
\hline Jaccard & 0.90 & 0.71 & 0.86 & - & 0.91 \\
\hline Dice & 0.94 & 0.80 & - & 0.88 & 0.95 \\
\hline
\end{tabular}

Table 3. Performance evaluation results recorded by proposed method and results published for four state-of-the-art algorithms on DRISHTI-GS dataset. (-) indicates result not available

\begin{tabular}{|c|c|c|c|c|c|c|}
\hline Metric & $\begin{array}{c}\text { Zilly et al. } \\
\mathbf{( 2 0 1 5 )}\end{array}$ & $\begin{array}{c}\text { Kumar et al. } \\
\mathbf{( 2 0 1 5 )}\end{array}$ & $\begin{array}{c}\text { Mohan et al. } \\
\mathbf{( 2 0 1 8 )}\end{array}$ & $\begin{array}{c}\text { Joshi et al. } \\
\mathbf{( 2 0 1 1 )}\end{array}$ & $\begin{array}{c}\text { Dey et al. } \\
\mathbf{( 2 0 1 9 )}\end{array}$ & Proposed \\
\hline Jaccard & 0.90 & 0.83 & 0.93 & - & - & 0.92 \\
\hline Dice & 0.95 & 0.91 & 0.96 & 0.96 & 0.96 & 0.96 \\
\hline
\end{tabular}

Table 4. Performance evaluation results recorded by proposed method and results published for eight state-of-the-art algorithms on MESSIDOR dataset

\begin{tabular}{|c|c|c|c|c|c|c|c|c|c|}
\hline Algorithm & $\begin{array}{c}\text { Morales } \\
\text { et al. } \\
\text { (2017) }\end{array}$ & $\begin{array}{c}\begin{array}{c}\text { Sigut et } \\
\text { al. }\end{array} \\
\text { (2017) }\end{array}$ & $\begin{array}{c}\text { Aquino } \\
\text { et al. } \\
(2010)\end{array}$ & $\begin{array}{c}\text { Dey } \\
\text { et al. } \\
(2019)\end{array}$ & $\begin{array}{c}\begin{array}{c}\text { Zilly et } \\
\text { al. }\end{array} \\
\text { (2017) }\end{array}$ & $\begin{array}{l}\text { Cheng } \\
\text { et al. } \\
(2013)\end{array}$ & $\begin{array}{c}\text { Kumar } \\
\text { et al. } \\
\text { (2015) }\end{array}$ & $\begin{array}{c}\text { Mohan } \\
\text { et al. } \\
\text { (2018) }\end{array}$ & Proposed \\
\hline Dice Score & 0.90 & 0.95 & 0.92 & 0.91 & 0.95 & 0.93 & 0.85 & 0.96 & 0.92 \\
\hline
\end{tabular}

Dice similarity score and Jaccard index were adopted as the performance evaluation metrics. Histogram distribution of Dice score recorded by the proposed method on IDRID, DRISHTI-GS and MESIDOR datasets are displayed in Figure 4a-c, respectively. Table 2-4 display the performance metrics recorded from the proposed method and prior contributions on IDRID, DRISHTI-GS and MESSIDOR datasets, respectively. The proposed method recorded mean Dice scores of $0.95,0.96$ and 0.92 on IDRID, DRISHTI-GS and MESSIDOR datasets, respectively. It recorded Dice score greater than 0.8 on 100 percent of the IDRID and DRISHTI-GS datasets with mean standard deviation Dice score of 0.02 and 0.06 , respectively. Corresponding performance on the MESSIDOR dataset is 90 percent and mean standard deviation Dice score of 0.09 . The proposed method failed to segment less than 1 percent of test data from MESSIDOR dataset (see Figure 4c). The proposed method is unique in comparison to current methods because it enhances the performance of k-means clustering technique by means of the clustering tree. Also, it 
exploits the noise reduction capability of the mode filter to reduce occlusions by blood vessels within the optic disc region. Furthermore, segmentation accuracy is optimized by feature extraction and pooling from the two color channels. Based on the formulation and performance of our proposed method as well as the performance evaluation results that was published from prior contributions, it is reasonable to state that the proposed method is comparable to current state-of-the-art algorithms.

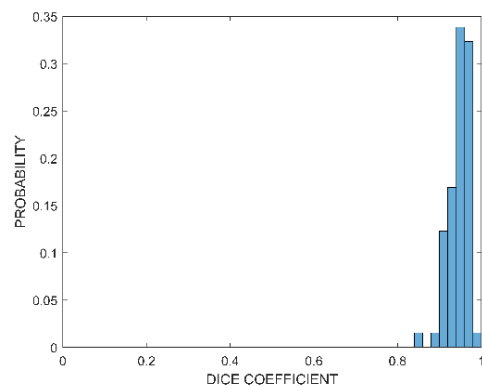

(a)

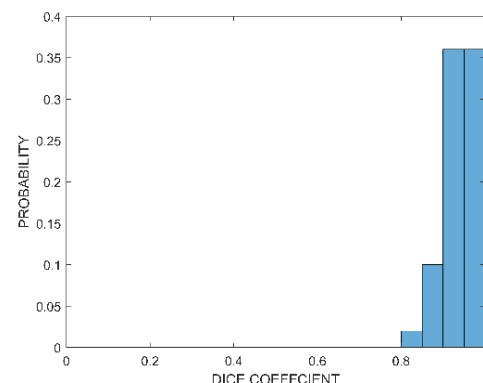

(b)

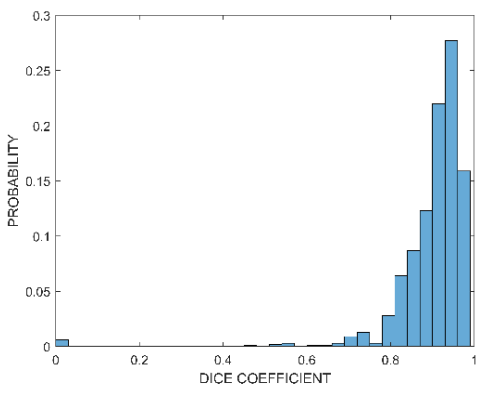

(c)

Figure 4. Histogram distribution of Dice scores recorded by the proposed method on (a) IDRID, (b) DRISHTI-GS and (c) MESSIDOR datasets

\section{CONCLUSION}

In this paper, we propose a new optic disc segmentation method that applies the k-means algorithm to generate a clustering tree. The clustering tree does not require the user to specify predefined number of clusters, thus it overcomes the major limitations of the k-means algorithm in automatic segmentation. The clustering tree generates low-level feature image from each color channel. The features were extracted and pooled from two color channels to optimize segmentation. The proposed method also exploits the noise reduction capability of the mode filter to suppress occlusions by blood vessels. Performance evaluation on three publicly available datasets shows that the proposed method is comparable to some state-of-the -art algorithms.

\section{ACKNOWLEDGEMENT}

This work is supported by the European Research Consortium on Informatics and Mathematics (ERCIM).

\section{REFERENCES}

Aquino, A., Gegúndez-Arias, M. E., \& Marín, D. (2010). Detecting the optic disc boundary in digital fundus images using morphological, edge detection, and feature extraction techniques. IEEE transactions on medical imaging, 29(11), 1860-1869.

Baid, U., Baheti, B., Dutande, P., \& Talbar, S. (2019, October). Detection of Pathological Myopia and Optic Disc Segmentation with Deep Convolutional Neural Networks. In TENCON 2019-2019 IEEE Region 10 Conference (TENCON) (pp. 1345-1350). IEEE.

Chalakkal, R. J., Abdulla, W. H., \& Thulaseedharan, S. S. (2018). Automatic detection and segmentation of optic disc and fovea in retinal images. IET Image Processing, 12(11), 2100-2110.

Cheng, J., Liu, J., Xu, Y., Yin, F., Wong, D. W. K., Tan, N. M., ... \& Wong, T. Y. (2013). Superpixel classification based optic disc and optic cup segmentation for glaucoma screening. IEEE transactions on medical imaging, 32(6), 1019-1032. 
Dey, S., Tahiliani, K., Kumar, J. H., Pediredla, A. K., \& Seelamantula, C. S. (2019, May). Automatic Segmentation of Optic Disc Using Affine Snakes in Gradient Vector Field. In ICASSP 2019-2019 IEEE International Conference on Acoustics, Speech and Signal Processing (ICASSP) (pp. 1204-1208). IEEE.

Huelva, U. (2012). Expert system for early automated detection of OD by analysis of digital retinal images (http://www.uhu.es/retinopathy).

Joshi, G. D., Sivaswamy, J., \& Krishnadas, S. R. (2011). Optic disk and cup segmentation from monocular color retinal images for glaucoma assessment. IEEE transactions on medical imaging, 30(6), 1192-1205.

Klein, J. C., Menard, M., Cazuguel, G., Fernandez-Maloigne, C., Shaefer, G., Gain, P., ... \& Charton, B. (2016). Methods to evaluate segmentation and indexing techniques in the field of retinal ophthalmology (MESSIDOR). Ecole des Mines de Paris.

Kumar, JR Harish, Aditya Kumar Pediredla, and Chandra Sekhar Seelamantula. "Active discs for automated optic disc segmentation." In 2015 IEEE global conference on signal and information processing (GlobalSIP), pp. 225-229. IEEE, 2015.

Lim, G., Cheng, Y., Hsu, W., \& Lee, M. L. (2015, November). Integrated optic disc and cup segmentation with deep learning. In 2015 IEEE 27th International Conference on Tools with Artificial Intelligence (ICTAI) (pp. 162-169). IEEE.

Lloyd, S. (1982). Least squares quantization in PCM. IEEE transactions on information theory, 28(2), 129-137.

Lowell, J., Hunter, A., Steel, D., Basu, A., Ryder, R., Fletcher, E., \& Kennedy, L. (2004). Optic nerve head segmentation. IEEE Transactions on medical Imaging, 23(2), 256-264.

Marrugo, A. G., Vargas, R., Chirino, M., \& Millán, M. S. (2015, December). On the illumination compensation of retinal images by means of the bidimensional empirical mode decomposition. In 11th International Symposium on Medical Information Processing and Analysis (Vol. 9681, p. 96810D). International Society for Optics and Photonics.

Mohan, D., Kumar, J. H., \& Seelamantula, C. S. (2018, October). High-performance optic disc segmentation using convolutional neural networks. In 2018 25th IEEE International Conference on Image Processing (ICIP) (pp. 4038-4042). IEEE.

Morales, S., Naranjo, V., Angulo, J., \& Alcañiz, M. (2013). Automatic detection of optic disc based on PCA and mathematical morphology. IEEE transactions on medical imaging, 32(4), 786-796.

Osadebey, M., Pedersen, M., Arnold, D., \& Wendel-Mitoraj, K. (2019). Local Indicators of Spatial Autocorrelation (LISA): Application to Blind Noise-Based Perceptual Quality Metric Index for Magnetic Resonance Images. Journal of Imaging, 5(1), 20.

Pohlen, T., Hermans, A., Mathias, M., \& Leibe, B. (2017). Full-resolution residual networks for semantic segmentation in street scenes. In Proceedings of the IEEE Conference on Computer Vision and Pattern Recognition (pp. 4151-4160).

Porwal, P., Pachade, S., Kadethankar, A., Joshi, A., Patwardhan, V., Kamble, R., ... \& Meriaudeau, F. (2018, August). Automatic segmentation of optic disc by gradient minimization based approach. In 2018 International Conference on Intelligent and Advanced System (ICIAS) (pp. 1-5). IEEE.

Porwal, P., Pachade, S., Kamble, R., Kokare, M., Deshmukh, G., Sahasrabuddhe, V., \& Meriaudeau, F. (2018). Indian diabetic retinopathy image dataset (IDRiD): a database for diabetic retinopathy screening research. Data, 3(3), 25.

Roychowdhury, S., Koozekanani, D. D., Kuchinka, S. N., \& Parhi, K. K. (2015). Optic disc boundary and vessel origin segmentation of fundus images. IEEE journal of biomedical and health informatics, 20(6), 1562-1574

Sigut, J., Nunez, O., Fumero, F., Gonzalez, M., \& Arnay, R. (2017). Contrast based circular approximation for accurate and robust optic disc segmentation in retinal images. PeerJ, 5, e3763.

Sivaswamy, J., Krishnadas, S. R., Joshi, G. D., Jain, M., \& Tabish, A. U. S. (2014, April). Drishti-gs: Retinal image dataset for optic nerve head (onh) segmentation. In 2014 IEEE 11th international symposium on biomedical imaging (ISBI) (pp. 53-56). IEEE.

Zappia, L., \& Oshlack, A. (2018). Clustering trees: a visualization for evaluating clusterings at multiple resolutions. GigaScience, 7(7), giy083.

Zilly, J., Buhmann, J. M., \& Mahapatra, D. (2017). Glaucoma detection using entropy sampling and ensemble learning for automatic optic cup and disc segmentation. Computerized Medical Imaging and Graphics, 55, $28-41$.

Zilly, J. G., Buhmann, J. M., \& Mahapatra, D. (2015, October). Boosting convolutional filters with entropy sampling for optic cup and disc image segmentation from fundus images. In International workshop on machine learning in medical imaging (pp. 136-143). Springer, Cham. 